\title{
Controversies of radiotherapy in human epidermal growth factor receptor (HER)-2 positive breast cancer patients
}

\author{
Iveta Kolarova ${ }^{\mathrm{a}, \mathrm{b}}$, Bohuslav Melichar ${ }^{\mathrm{a}, \mathrm{c}, \mathrm{d}}$, Jaroslav Vanasek ${ }^{\mathrm{b}, \mathrm{e}}$, Ales Ryska ${ }^{\mathrm{f}}$, Katerina Horackova ${ }^{\mathrm{b}}$, Jiri Petera ${ }^{\mathrm{a}, \mathrm{d}},{ }_{\text {, Milan Vosmik }}^{\mathrm{a}, \mathrm{d}}$, \\ Igor Sirak ${ }^{\mathrm{a}, \mathrm{d}}$, Martin Dolezel ${ }^{\mathrm{c}, \mathrm{g}}$
}

Tumor biology plays a crucial role in the systemic treatment, specifically in HER2-positive tumors. Distinct biological behavior of breast cancer subtypes is associated with different rates of locoregional recurrence (LRR). HER2- positive breast cancer patients treated with surgery in combination with radiation, without trastuzumab have poor outcome, including high LRR. The efficacy of radiotherapy in HER-2-positive breast cancer appears to be associated with the expression of estrogen receptors. In patients with HER-2-positive breast cancer, studies conducted before the introduction of trastuzumab indicated higher benefit of adjuvant radiation in patients with hormone receptor-positive tumors compared to patients with tumors not expressing hormone receptors. The introduction of agents targeting HER- 2 has transformed the management of these patients, resulting in improved outcomes. The data of clinical studies show that the administration of trastuzumab as part of a multimodality approach (with radiation based on standard guidelines) results in improved outcomes, including lower locoregional recurrence. The risk of cardiac toxicity associated with radiation to the heart and administration of potential cardiotoxic trastuzumab is not clear. In patients treated concomitantly with regional lymph node irradiation and anti-HER-2 agents after prior anthracycline-based chemotherapy minimizing the dose to the myocardium, e.g. respiratory gating or proton beam radiotherapy, have been suggested.

Key words: breast cancer, radiation therapy, HER-2, locoregional recurrence, trastuzumab

Received: October 8, 2020; Revised: November 26, 2020; Accepted: January 15, 2021; Available online: February 4, 2021

https://doi.org/10.5507/bp.2021.007

(c) 2021 The Authors; https://creativecommons.org/licenses/by/4.0/

${ }^{a}$ Department of Oncology and Radiotherapy, University Hospital Hradec Kralove, Czech Republic

${ }^{b}$ Faculty of Health Studies, Pardubice University, Pardubice, Czech Republic

'Department of Oncology, Faculty of Medicine and Dentistry, Palacky University and University Hospital Olomouc, Czech Republic

${ }^{d}$ Department of Oncology and Radiotherapy, Faculty of Medicine in Hradec Kralove, Charles University, Czech Republic

encology Centre, Multiscan, Pardubice, Czech Republic

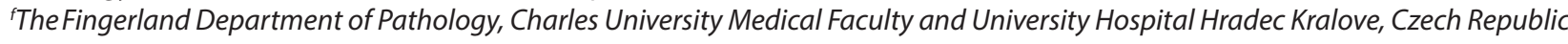
${ }^{9}$ Department of Oncology, First Faculty of Medicine, Charles University, Prague, Czech Republic

Corresponding author: Martin Dolezel, e-mail: dolezelm@email.cz

\section{INTRODUCTION}

Historically, the assessment of prognosis of patients with newly diagnosed breast cancer, including therapeutic consequences, was based on information reflecting the tumor size and extent of disease spread rather than on tumor biology. In contrast, breast cancer subtypes that are associated with differences in tumor biology, prognosis and response to therapy are playing an increasing role in therapeutic decisions. Different biological behavior of breast cancer subtypes is also associated with variations of locoregional recurrence (LRR) rates both in patients treated with breast-conserving surgery (BCS) and adjuvant radiation and in patients after radical mastectomy without adjuvant radiation ${ }^{1}$.

The information on tumor biology has fundamental implications for systemic therapy, in particular in patients with HER-2-positive tumors. The introduction of agents targeting HER-2 has transformed the management of these patients, resulting in improved outcomes. Some biological tumor characteristics of the tumor, including grade, proliferation rate, expression of estrogen receptor
(ER), progesterone receptor (PR) and HER-2 and prognostic gene expression panels were therefore included in the Eighth Edition of the American Joint Committee on Cancer (AJCC) Staging Manual. To maintain continuity and utilization across the globe anatomical staging system was kept. The prognostic value of commercial gene profile tests was endorsed. Biomarkers reflecting the tumor biology and Oncotype Dx recurrence score are considered to be sufficiently robust predictors of outcome to influence management decisions ${ }^{2}$.

Importantly, the new classification is limited to patients treated with primary surgery. A study in patients treated with neoadjuvant chemotherapy was performed in 2363 patients treated between 2005 and 2012 in M.D. Anderson Cancer Center staged either based on AJCC $8^{\text {th }}$ Edition or Neo-Bioscore (which is based on clinical and pathological stage, grade, estrogen receptor status and HER-2 expression and classifies patients with any breast cancer subptype according the disease-specific survival).

This study confirms the significance of biological biomarkers along with anatomical staging for the prognosis of breast cancer patients treated with neoadjuvant 
chemotherapy. Data indicate that biological factors are important determinants of prognosis and anatomical stage of AJCC $8^{\text {th }}$ Edition may be used in these patients. AJCC prognostic stage may be considered as comparator in the investigations aiming at the definition of optimal method of staging in breast cancer patients treated with neoadjuvant therapy ${ }^{3}$.

In patients with tumors expressing ER and no lymph node involvement, an association between Oncotype DX score (GenomicHealth, Redwood City, USA) and LRR risk was observed ${ }^{4}$. The association between the score obtained by this test based on the expression of 21 genes and LRR was studied in postmenopausal breast cancer patients with positive lymph nodes and tumors expressing ER or PR treated either with adjuvant chemotherapy followed by tamoxifen or with tamoxifen alone. Medium or high score was associated with significantly increased risk of LRR in the entire cohort as well as in patients treated with mastectomy who had no adjuvant radiation. The hazard ratio for LRR in patients with high recurrence score was 2.36 , with $95 \%$ confidence interval (CI) 1.02 $5.45(P=0.04)$. In the subgroup analysis of patients after mastectomy with 1 to 3 involved lymph nodes not treated with adjuvant radiation, LRR was observed in $1.5 \%$ of patients with low risk score and $11.1 \%$ patients with medium or high risk score $(P=0.051)\left(\right.$ ref. $\left.^{5}\right)$.

\section{Breast cancer subtypes and locoregional control}

As mentioned above, tumor biology is an important determinant of LRR risk. The significance of breast cancer subtypes before the introduction of targeted therapy was investigated in a Canadian study that included 2985 patients with early invasive breast cancer. Patients were classified based on the expression of ER, PR, Ki-67, HER-2, epidermal growth factor receptor (EGFR), and cytokeratin (CK) 5/6 into subgroups including luminal A, luminal B, luminal HER-2, HER2 enriched, basal-like and triple negative - non-basal. Risk of local and regional recurrence associated with the intrinsic subtypes was studied with multivariate Cox analysis after adjusting for standard clinical and pathological parameters. None of the patients was treated with trastuzumab in the neoadjuvant or adjuvant setting. After a median follow up of 12 years, 325 local recurrences and 227 regional lymph node recurrences were observed. Patients with luminal A tumors (ER or PR positive, HER-2-negative, Ki-67 < $14 \%$ ) had better prognosis and lowest risk of LRR. A significantly higher risk of LRR after BCS was observed in multivariate analysis in patients with HER-2 enriched and basal tumors. In patients after mastectomy luminal B, luminal HER-2, HER-2 enriched and basal subtypes were associated with significantly increased LRR rate. This leads to the conclusion that the determination of tumor subtype using immunohistochemistry may identify patients with higher risk of LRR. Luminal A tumors are associated with low and HER-2 enriched or basal with high risk of LRR (ref. ${ }^{1}$ ).

\section{Radiation therapy and HER-2 positivity}

HER-2 over-expression is one of the most important molecular biomarkers in breast cancer that is associated with proliferation rate, invasion and metastasis and response to radiation. Before the advent of targeted therapy, HER-2 over-expression predicted poor outcome. HER-2 is a member of a family of receptor tyrosine kinases that includes also EGFR/HER1, c-erbB2/HER2, HER3, HER4. HER-2 is a universal co-receptor for other protein of the family. HER-2 over-expression or amplification stimulates tumor growth, invasive phenotype and tumor cell survival through the activation of cell signaling pathways, in particular MAPK (Mitogen-activated protein kinase) a PI3k ( phosphatidylinositol 3-kinase) /akt (serine/threonine protein kinase) $\left(\right.$ ref. $\left.^{6}\right)$.

A humanized monoclonal antibody against HER-2 trastuzumab, introduced in late 1990s was the first approved targeted therapy in breast cancer. Improved outcomes, including prolongation of overall survival (OS), has been demonstrated after addition of trastuzumab to cytotoxic agents in patients with advanced/metastatic breast cancer as well as in the adjuvant or neoadjuvant setting ${ }^{7,8}$.

The spectacular activity of trastuzumab stimulated the development of other therapies targeting HER-2 including other antibodies (pertuzumab), receptor tyrosine kinase inhibitors (lapatinib and neratinib) and conjugates of monoclonal antibody to cytotoxic drug (trastuzumab emtansin). Current standard of systemic treatment of HER-2-positive breast cancer is based on the combination of anti-HER-2 drugs with cytotoxic or hormonal agents. The introduction of anti-HER-2 represents a paradigm of successful utilization of targeted therapy in medical oncology .

It has been observed that ionizing radiation can directly activate proteins of the EGFR family in tumor cells, and repeat irradiation by 2 Gy results in up-regulated EGFR expression in patients with HER-2 enriched subtype tumors, indicating that HER-2 positivity may be associated with the response to radiation ${ }^{10}$. This hypothesis is further supported by the data of clinical trials examining the efficacy of radiotherapy. The patients with HER-2 over-expressing tumors treated with mastectomy in combination with radiation, but no trastuzumab have poor outcome, including high LRR (ref., ${ }^{1,11}$ ). A retrospective study in HER-2-positive patients with negative lymph nodes treated with BCS and conventional whole breast irradiation has demonstrated a LRR rate after 3 years of $1 \%$ for patients treated with trastuzumab and $9 \%$ in patients not treated with trastuzumab ${ }^{12}$.

A number of studies have investigated the molecular mechanisms of resistance to radiation in HER-2-positive tumors. In vitro experiments demonstrate a radioresistance of HER-2-positive breast tumors mediated by the activation of NF-kappaB (nuclear factor kappa B) and PI3K / Akt pathways. The expression of HER-2 is further increased which is responsible for resistance to radiation. Higher resistance to radiation and increased recurrence rate after radiation is also associated with the presence 
of breast cancer stem cells ${ }^{11,13,14}$. Hou et al. ${ }^{15}$ described a mechanism of radiation resistance cause by activation of focal adhesive kinase (Fak) and epithelial/mesenchymal transformation. A crucial role in epithelial/mesenchymal transformation is played by $\beta$-catenin that can be detected in invasive and metastatic HER-2-positive tumors $^{16-18}$. Importantly, in vivo studies have confirmed clinical benefit of Fak pathway inhibition that plays a key role in increase radioresistance of HER-2-enriched subtype tumors ${ }^{10,15}$.

\section{The significance of hormone receptor expression in patients with HER-2-positive tumors not treated with trastuzumab}

A number of studies have indicated that the efficacy of radiotherapy in HER-2-positive breast cancer is dependent on the expression of ER. A Danish study randomized postmastectomy radiotherapy PMRT in patients with positive lymph nodes. In patients with HER-2 positive tumors after mastectomy increased radioresistance was observed in the pre-trastuzumab era. In particular, OS was not significantly improved after PMRT in patients with HER-2-positive tumors not expressing hormone receptors. The results indicated that the hormone receptor positivity was the most important predictor of efficacy of radiotherapy. Patients with HER-2-positive/ER-positive tumors had an absolute reduction of LRR rate at 10 years of $45 \%$ in contrast to $12 \%$ in patients with HER-2-positive/ERnegative tumors. After 10 years, only $3 \%$ of patients with HER-2-positive/ER-positive tumors experienced recurrence compared to $21 \%$ in patients with HER-2-positive/ ER-negative tumors ${ }^{19}$.

A meta-analysis based on data of 10801 patients enrolled in 17 randomized trials conducted before the introduction of trastuzumab also indicated a difference in response to adjuvant radiation in patients with HER-2positive breast cancer based on hormone receptor positivity with patients with hormone receptor-positive tumors deriving higher benefit compared to patients with tumors not expressing hormone receptors ${ }^{20}$.

\section{The significance of the introduction of trastuzumab}

Several randomized trials have demonstrated a significant effect of adjuvant trastuzumab on LRR rate in patients with HER-2 positive breast cancer. Radiotherapy was used in these trials based on current standards, i.e. in patients after BCS or in patients after mastectomy and additional risk factors ${ }^{21-26}$.

The significance of breast cancer subtypes for risk of LRR in the trastuzumab era was evaluated in a meta-analysis based on data of 11219 patients enrolled in 7 trials. LRR rate varies across breast cancer subtypes with the lowest rate in luminal tumors and highest rates in triple negative breast cancer. Low LRR rate after the introduction of anti-HER-2 therapy is a reflection of therapeutic advances. The LRR rate in the whole population was $3.44 \%$. The lowest (1.7\%) and highest (7.4\%) LRR rates were observed in patients with luminal A and triple negative breast cancer subtypes, respectively. The risk of LRR was significantly lower in patients with luminal A compared to luminal B tumors, with odds ratio (OR) of 0.54 ; 95\% confidence intervals $(\mathrm{CI}) 0.38-0.76 ; P<0.0004$, HER-2-positive tumors (OR 0.32; 95\% CI 0.24-0.45; $P<0.0001$ ) and triple negative breast cancer (OR 0.25; 95\% CI 0.19-0.32; $P<0.0001)$. A significant difference in LRR was observed between luminal B and HER-2positive tumors (OR 0.61; 95\% CI 0.41-0.89; $P=0.0145$ ). Compared to triple negative breast cancer patients with HER-2-positive tumors exhibited a trend of lower risk of LRR of borderline statistical significance (OR 0.75; 95\% CI 0.55-1.03; $P=0.0933$ ) (ref. ${ }^{27}$ ).

A number of randomized trials in patients with HER2-positive tumors demonstrated LRR rates ranging between 2 and $5 \%$ in patients treated with trastuzumab and 4 to $10 \%$ without trastuzumab indicating superior locoregional control with the combination of radiotherapy and trastuzumab. This effect is even more pronounced in patients with advanced HER-2-positive breast cancer who underwent PMRT (ref. ${ }^{28}$ ).

A single center analysis from Memorial SloanKettering Cancer Center reported LRR rate at 5 years in only $2 \%$ of stage I to III patients treated with trastuzumab. The LRR rate was higher in patients not treated with adjuvant radiation $(5 \% v 0 \% ; P=0.06)\left(\right.$ ref. $\left.^{29}\right)$.

A recent study from Sweden analyzed the distribution of LRR based on the radiotherapy technique and breast cancer subtypes ${ }^{30}$. A total of 923 patients treated with postoperative locoregional radiation therapy between 2004 and 2008 analyzed. Cumulative LRR incidence after 10 years was $7.1 \%$ (95\% CI 5.5-9.1). LRR was observed in 57 out of 923 patients (30 cases of local recurrence and 30 cases of regional recurrence, including 3 patients with both local and regional recurrence). Most cases of LRR were localized in fully (56\%) or marginally (26\%) irradiated areas. The most common site of regional recurrence outside of irradiated field was located cranially in the supraclavicular fossa. ER-negative or HER-2-positive tumors constituted $75 \%$ of recurrent lesions in the radiation field, but only $45 \%$ of cases in the marginally irradiated or not irradiated areas. Compared to patients with ER-positive tumors, LRR risk was higher in patients with ER-negative (hazard ratio (HR) 4.6; $P<0.001 ; 95 \% \mathrm{CI}$, 2.5-8.4) or HER-2-positive (HR 2.4; $P=0.007$; 95\% CI, 1.34.7) breast cancer.

Data from National Comprehensive Cancer Network indicate that administration of radiotherapy as part of current therapeutic approaches was significantly independently associated with improved LRR rate (HR 0.12; $P=0.006$ ) $\left(\right.$ ref. $\left.^{14}\right)$. These data indicate the efficacy of the combination of radiotherapy and trastuzumab in preventing LRR. The omission of radiotherapy in standard indications is not justified in the light of these findings.

\section{The significance of hormone receptor expression in patients with HER-2-positive tumors treated with trastu- zumab}

In the current clinical practice the administration of systemic therapy is guided by the presence of hormone 
receptors (ER and PR) and HER-2 over-expression. Hormone receptor expression in tumor cells is observed approximately in $75 \%$ of cases, while HER-2 amplification is reported to be present in $15-20 \%$ of patients. Tumors with both hormone receptor expression and HER-2 overexpression, sometimes called triple positive breast cancer (TPBC), represent about a half of HER-2 positive tumors ${ }^{9}$. Complex interaction between the HER-2 and hormone receptor signaling is thought to occur in these cases, and the activation of hormone receptor associated pathways is thought to be one of the mechanisms of resistance to anti-HER-2-therapy. Tumors with high degree of HER-2 over-expression are usually characterized by absent of low hormone receptor expression ${ }^{31}$.

The significance of hormone receptor expression for the efficacy of the combination of trastuzumab with radiotherapy remains a matter of debate. In a study of 1000 patients younger than 35 years, cases of LRR after trastuzumab were observed in patients with ER-negative, HER-2 positive tumors and none among patients with ER-positive, HER-2 positive tumors. Radiotherapy was administered according the standard guidelines ${ }^{32}$.

Another study investigating the significance of ER positivity in patients with HER-2 positive tumors demonstrated no difference in LRR rate with or without the administration of trastuzumab. The LRR rates in patients with ER-positive, HER-2 positive and ER-negative, HER-2 negative patients were comparable in this study ${ }^{33}$.

The role of ER expression in the response of HER2-positive breast cancer to radiation was investigated in a retrospective analysis of HERA trial in patients with $\mathrm{N} 1$ tumors (1-3 involved lymph nodes) (ref. ${ }^{34}$ ). The administration of adjuvant radiotherapy resulted in significantly lower LRR risk in patients with ER-positive tumors, in agreement with other studies demonstrating significant benefit of PMRT in patients with ER-positive HER-2 positive tumors compared to ER-negative, HER-2-positive breast cancer ${ }^{14,19,33,35}$. In vitro experiments investigated the effect of ER expression on radiosensitivity. The transduction of ER resulted in delayed DNA repair, accentuated apoptosis after irradiation and higher proportion of cells in the radiosensitive phases of the cell cycle $(\mathrm{G} 2 / \mathrm{M})$ (ref. ${ }^{36}$ ).

Favorable results of therapy in TPBC are explained by the availability of multiple treatment options, i.e. surgery, radiotherapy, hormonal therapy, targeted therapy as well as cytotoxic chemotherapy ${ }^{37}$.

In a study analyzing OS and breast cancer-specific survival of 166054 breast cancer patients in the SEER (The Surveillance, Epidemiology, and End Results Program of the National Cancer Institute) database OS was significantly different among different subtypes at every stage $(P<0.0001)$. The 3 -year OS among stage I patients was highest in patients with hormone receptor-positive, HER-2-negative tumors (97.2\%) while hormone receptor-positive, HER-2-positive patients had best 3-year OS among stage II (94.5\%), stage III (87.8\%) and stage IV (54.8\%) patients. A multivariate analysis that included age, race, tumor grade, histology and family status confirmed these results. The analysis demonstrated marked differences of OS and breast cancer-specific survival rates at each stage that retained significance in the multivariate model. Moreover, the differences in overall and breast cancer-specific survival rate are substantially increasing with advancing stage ${ }^{38}$.

\section{Significance of pathological complete response after neoadjuvant therapy}

The efficacy of neoadjuvant therapy is another factor determining the locoregional control. In one study LRR rate was significantly decreased in patients with HER-2positive tumors in case of pathological complete response after neoadjuvant therapy regimens with trastuzumab $(2.6 \% v 13.3 \%)\left(\right.$ ref. $\left.^{39}\right)$. In patients with HER-2-positive tumors and pathological complete response LRR rates are similar to patients with luminal A tumors, but LRR rate is high in patients with less favorable pathological response ${ }^{14,39}$

On the other hand, other studies question the significance of pathological complete response in patients with TPBC. A number of prospective trials have established a significantly lower pathological complete response rate in TPBC compared to patients with hormone receptornegative, HER-2-positive tumors. Interestingly, the lower pathological complete response rate does not seem to have an effect on the generally favorable prognosis of TPBC $\left(\right.$ ref. $\left.^{40}\right)$. It has even been speculated whether the administration of neoadjuvant therapy regimens with cytotoxic chemotherapy in patients with early TPBC with favorable prognosis is not an overtreatment ${ }^{41}$.

\section{Radiation therapy approaches in combination with targeted therapy}

Irradiation of regional lymph nodes in patients with HER-2-positive breast cancer treated with trastuzumab remains a disputed topic. In a retrospective analysis of 1664 patients with HER-2-positive tumors, BCS and positive lymph nodes in the ALTTO trial, regional lymph node irradiation was performed in 878 patients with more advanced disease and higher number of involved lymph nodes. After a median follow up of 4.5 years no significant difference in disease-free survival was observed in patients with or without regional lymph node irradiation $(84.3 \% v 88.3 \%)$. The problem in the interpretation of this analysis is associated with the use of different regimens of anti-HER-2 therapy with trastuzumab, lapatinib in various sequences and the fact that regional lymph node irradiation was defined as radiotherapy to any lymph node region without further details on dose and field distribution. However, it could be speculated whether regional lymph node irradiation did not result in lower number of recurrences in patients with high LRR risk ${ }^{42}$.

The risk of cardiac toxicity associated with the radiation to the heart and administration of potential cardiotoxic trastuzumab is also not clarified. Importantly, in an analysis of 1503 patients treated with adjuvant radiation in NCCTG N9831 randomized trial no increase of cardiac toxicity was observed, not even in patients treated with concomitant trastuzumab and radiotherapy ${ }^{43}$. 
There are limited data on cardiac toxicity of concomitant trastuzumab and radiotherapy in patients undergoing internal mammary lymph node irradiation that results in increased radiation dose to the heart and higher risk of cardiotoxicity. In a prospective French study of 308 patients with HER-2-positive breast cancer radiotherapy to internal mammary lymph nodes was administered in 227 cases $(73.7 \%)$. A decrease of left ventricular ejection fraction was observed in $8.4 \%$ patients, but was of grade 2 or higher in $2.9 \%$, which is similar to the rates reported from large randomized trials of trastuzumab administered with anthracycline-containing chemotherapy ${ }^{44}$. In patients treated concomitantly with regional lymph node irradiation and anti-HER-2 agents after prior anthracycline-based chemotherapy techniques minimizing dose to the myocardium, e.g. respiratory gating or proton beam radiotherapy have been suggested.

Radiation dose reduction or elimination using boost to tumor bed represents another option for reducing toxicity. In the pre-trastuzumab era LRR rate was dependent on ER expression. Patients with HER-2-positive tumors expressing ER have very low LRR rate, and, similarly to patients with HER-2- negative luminal A tumors, omission of the boost to tumor bed or dose reduction may be considered. The introduction of other drugs into the therapy of HER-2 positive and ER positive breast cancers also has a beneficial effect. For example, the results of a study with neratinib in extended adjuvant showed a significant improvement in invasive disease free survival ${ }^{45}$. A lower risk of relapses may lead to a reduction in the indications for radiation therapy, or a change in the radiotherapy technique in the sense of reducing the irradiated volume. Considerations concerning a complete elimination of adjuvant radiation from the treatment algorithm are, however, still premature.

A number of experimental studies have demonstrated a radiosensitizing effect of trastuzumab on tumor cells. It is also hypothesized that concomitant administration of trastuzumab increases the efficacy of adjuvant radiation in clinical practice, which is supported by lowering of LRR rate by the administration of trastuzumab. However, further research is needed to determine the optimal use of trastuzumab as a radiosensitizing agent ${ }^{28}$.

The use of boost in patients with ER-negative, HER2-positive tumors treated with adjuvant trastuzumab and radiotherapy remains disputed. In the absence of relevant data, utilization of boost could be considered based on the data from studies demonstrating the benefit in an unselected patient population ${ }^{46}$.

Current standard methods of adjuvant radiation therapy are characterized by high efficacy as well as an excellent safety profile and tolerance ${ }^{47}$.

\section{CONCLUSION}

Histopathological classification and gene expression profiling can be used to classify breast cancer into different subtypes. This classification reflects the heterogeneous nature of tumor biology, including different LRR rate. Molecular signatures are a basis for stratifying BC patients for systemic therapies. Unfortunately, there are currently no clinically-validated signatures that can reliably classify breast cancers according to radiosensitivity.

However, significant improvement of radiotherapy was achieved in patients with HER2-positive tumors. In the past HER-2-positive breast cancer patients treated with mastectomy and radiation generally had poor outcomes, including high LRR rate. The advent of trastuzumab has had profound effect on the biology, natural course of disease and management of HER-2-positive breast cancer, including the LRR.

Further advancement in the therapy of HER-2-positive breast cancer is associated with the development of therapeutic guidelines based on multidisciplinary consensus. Maximal effort should be dedicated to integrate each fundamental treatment modality, i.e. surgery, pharmacotherapy and radiotherapy, into the therapeutic algorithms and protocols. Improved knowledge about biological behavior of breast cancer subtypes will allow a further refinement of indications and target volumes and guide the employment of modern radiotherapy techniques.

\section{Search strategy and selection criteria}

Our research strategy was aimed at evaluating studies on the role of radiotherapy in patients with HER2-positive breast cancer. Scientific articles from 2000 to 2020 were searched using the PubMed and Web of Science databases. All searches were up to date as of June 2020. The search terms used included "breast cancer subtypes", "HER-2 positivity”, "estrogen receptor", "anti-HER-2 therapy“, "locoregional recurrence" and "survival”. Only English language papers were reviewed.

Author contributions: JV, BM: manuscript writing; AR, MV and MD: manuscript revision. IK: literature search, manuscript writing and final approval; AR: written English correction, MD, JP, MV, IS: critical reading, final approval, KH: literature search.

Conflict of interest statement: The authors state that there are no conflicts of interest regarding the publication of this article.

\section{REFERENCES}

1. Voduc KD, Cheang MC, Tyldesley S, Gelmon K, Nielsen TO, Kennecke $\mathrm{H}$. Breast cancer subtypes and the risk of local and regional relapse. J Clin Oncol 2010;28(10):1684-91.

2. Giuliano AE, Connolly JL, Edge SB, Mittendorf EA, Rugo HS, Solin LJ, Weaver DL, Winchester DJ,Hortobagyi GN. Breast cancer-major changes in the American Joint Committee on Cancer eighth edition cancer staging manual. CA Cancer J Clin 2017; 67(4):290-303.

3. Yi M, Lin H, Bedrosian I, Shen Y, Hunt K, Chavez-MacGregor M, King T, Mittendorf E. Staging for Breast Cancer Patients Receiving Neoadjuvant Chemotherapy: Utility of Incorporating Biologic Factors. Ann Surg Oncol 2020;27:359-66.

4. Mamounas EP, Tang G, Fisher B, Paik S, Shak S, Costantino JP, Watson D, Geyer CE Jr, Wickerham DL, Wolmark N. Association between the 21- gene recurrence score assay and risk of locoregional recurrence in node-negative, estrogen receptor-positive breast cancer: Results from NSABP B-14 and NSABP B-20. J Clin Oncol 2010;28:1677-83.

5. Woodward WA, Barlow WE, Jagsi R, Buchholz TA, Shak S, Baehner 
F, Whelan TJ, Davidson NE, Ingle JN, King TA, Ravdin PM, Osborne CK, Tripathy D, Livingston RB, Gralow JR, Hortobagyi GN, Hayes DF, Albain KS. Association Between 21-Gene Assay Recurrence Score and Locoregional Recurrence Rates in Patients With Node-Positive Breast Cancer. JAMA Oncol 2020;6(4):505-11. doi: 10.1001/jamaoncol.2019.5559.

6. Schettini F, Buono G, Cardalesi C, Desideri I, De Placido S, Del Mastro L. Hormone receptor/human epidermal growth factor receptor 2-positive breast cancer: where we are now and where we are going. Cancer Treat Rev 2016;46:20-26.

7. Romond EH, Perez EA, Bryant J, Suman VJ, Geyer CE Jr, Davidson NE, Tan-Chiu E, Martino S, Paik S, Kaufman PA, Swain SM, Pisansky TM, Fehrenbacher L, Kutteh LA, Vogel VG, Visscher DW, Yothers G, Jenkins RB, Brown AM, Dakhil SR, Mamounas EP, Lingle WL, Klein PM, Ingle $\mathrm{JN}$, Wolmark N. Trastuzumab plus adjuvant chemotherapy for operable HER2-positive breast cancer. N Engl J Med 2005;353(16):167384.

8. Smith I, Procter M, Gelber RD, Guillaume S, Feyereislova A, Dowsett M, Goldhirsch A, Untch M, Mariani G, Baselga J, Kaufmann M, Cameron D, Bell R, Bergh J, Coleman R, Wardley A, Harbeck N, Lopez RI, Mallmann P, Gelmon K, Wilcken N, Wist E, Sánchez Rovira P, Piccart-Gebhart MJ. 2-year follow-up of trastuzumab after adjuvant chemotherapy in HER2-positive breast cancer: a randomized controlled trial. Lancet 2007;369:29-36.

9. Larionov AA. Current Therapies for Human Epidermal Growth Factor Receptor 2-Positive Metastatic Breast Cancer Patients. Front Onco 2018;8:89.

10. He L, Lv Y, Song Y, Zhang B. The prognosis comparison of different molecular subtypes of breast tumors after radiotherapy and the intrinsic reasons for their distinct radiosensitivity. Cancer Manag Res 2019;11:5765-75.

11. Lowery AJ, Kell MR, Glynn RW, Kerin MJ, Sweeney KJ. Locoregional recurrence after breast cancer surgery: a systematic review by receptor phenotype. BreastCancer Res Treat 2012;133(3):831-41.

12. Kiess AP, McArthur HL, Mahoney K, Patil S, Morris PG, Ho A, Hudis, CA, Mc Cormick B. Adjuvant trastuzumab reduces locoregional recurrence in women who receive breast-conservation therapy for lymph node-negative, human epidermal growth factor receptor 2-positive breast cancer. Cancer 2012;118(8):1982-88.

13. Garvin S, Vikhe Patil E, Arnesson LG, Oda H, Hedayati E, Lindström $A$, Shabo I.Differences in intra-tumoral macrophage infiltration and radiotherapy response among intrinsic subtypes in pT1-T2 breast cancers treated with breast-conserving surgery. Virchows Arch 2019;475(2):151-62.

14. Tseng $Y D$, Uno $H$, Hughes $M E$, Niland $J C$, Wong $Y N$, Theriault $R$, Blitzblau RC, Moy B, Breslin T, Edge SB, Hassett MJ, Punglia RS Biological subtype predicts risk of locoregional recurrence after mastectomy and impact of postmastectomy radiation in a large national database. Int J Radiat Oncol Biol Phys 2015;93(3):622-30.

15. Hou J., Zhou Z., Chen X., Zhao R., Yang Z., Wei N., Ni Q., Feng Y., Yu $X .$, Ma J., Guo X. HER2 reduces breast cancer radiosensitivity by activating focal adhesion kinase in vitro and in vivo. Oncotarget 2016;7:45186-98.

16. Duru N, Fan M, Candas D, Menaa C, Liu HC, Nantajit D, Wen Y, Xiao K, Eldridge A, Chromy B A, Li S, Spitz DR, Lam K, Wicha MS, Li JJ.HER2associated radioresistance of breast cancer stem cells isolated from HER2-negative breast cancer cells. ClinCancer Res 2012;18(24):663447.

17. Giordano A, Gao H, Anfossi S, Cohen E, Mego M, Lee BN, Tin S, De Laurentiis M, Parker CA, Alvarez RH, Valero V, Ueno NT, De Placido S, Mani SA, Esteva FJ, Cristofanilli M, Reuben JM. Epithelialmesenchymal transition and stem cell markers in patients with HER2-positive metastatic breast cancer. Mol Cancer Ther 2012;11(11):2526-34. doi: 10.1158/1535-7163.MCT-12-0460

18. Arias-Romero LE, Villamar-Cruz O, Huang $M$, Hoeflich $K P$, Chernoff J. Pak1 kinase links ErbB2 to $\beta$-catenin in transformation of breast epithelial cells. Cancer Res 2013;73(12):3671-82.

19. Kyndi M, Sørensen FB, Knudsen H, Flemming, Overgaard M, Nielsen HM, Overgaard J. Estrogen receptor, progesterone receptor, HER-2, and response to postmastectomy radiotherapy in high-risk breast cancer: The Danish Breast Cancer Cooperative Group. J Clin Oncol 2008;26:1419-26.

20. Early BreastCancerTrialists' Collaborative Group, Darby S, McGale $P$, Correa C, Taylor C, Arriagada R, Clarke M, Cutter D, Davies C, Ewertz
M, Godwin J, Gray R, Pierce L, Whelan T, Wang Y, Peto R. Effect of radiotherapy after breast-conserving surgery on 10-year recurrence and 15-year breast cancer death: meta-analysis of individual patient data for 10,801 women in 17 randomised trials. Lancet 2011;378:1707-16.

21. Perez E, Suman V, Davidson N, Gralow J,Kaufman P, Ingle J,Dakhi S, Zujewski J, Pisansky T, Jenkins R. Results of chemotherapy alone, with sequential or concurrent addition of 52 weeks of trastuzumab in the NCCTG N9831 HER2-positive adjuvant breast cancer trial. Cancer Res 2009;69:80.

22. Slamon DJ, Leyland-Jones B, Shak S, Fuchs $H$, Paton V, Bajamonde A, Fleming T, Eiermann W, Wolter J, Pegram M, Baselga J, Norton L. Use of chemotherapy plus a monoclonal antibody against HER2 for metastatic breast cancer that overexpresses HER2. N Engl J Med 2001;344(11):783-92.

23. Joensuu H1, Bono P, Kataja V, Alanko T, Kokko R, Asola R, Utriainen T, Turpeenniemi-Hujanen T, Jyrkkiö S, Möykkynen K, Helle L, Ingalsuo $S$, Pajunen M, Huusko M, Salminen T, Auvinen P, Leinonen $H$, Leinonen M, Isola J, Kellokumpu-Lehtinen PL. Fluorouracil, epirubicin, and cyclophosphamide with either docetaxel or vinorelbine, with or without trastuzumab, as adjuvant treatments of breast cancer: final results of the FinHer Trial. J Clin Oncol 2009;27(34):5685-92.

24. Gianni L, Eiermann W, Semiglazov V, Manikhas A, Lluch A, Tjulandin S, Zambetti M, Vazquez F, Byakhow M, Lichinitser M, Climent MA, Ciruelos E, Ojeda B, Mansutti M, Bozhok A, Baronio R, Feyereislova A, Barton C, Valagussa P, Baselga J. Neoadjuvant chemotherapy with trastuzumab followed by adjuvant trastuzumab versus neoadjuvant chemotherapy alone, in patients with HER2-positive locally advanced breast cancer (the NOAH trial): a randomised controlled superiority trial with a parallel HER2-negative cohort. Lancet 2010;375:377-84

25. Romond EH, Perez EA, Bryant J, Suman VJ, Geyer CE Jr, Davidson NE, Tan-Chiu E, Martino S, Paik S, Kaufman PA, Swain SM, Pisansky TM, Fehrenbacher L, Kutteh LA, Vogel VG, Visscher DW, Yothers G, Jenkins RB, Brown AM, Dakhil SR, Mamounas EP, Lingle WL, Klein PM, Ingle JN, Wolmark N. Trastuzumab plus adjuvant chemotherapy for operable HER2-positive breast cancer. N Engl J Med 2005;353(16):167384.

26. Spielmann $M$, Roché $H$, Delozier $T$, Canon JL, Romieu G, Bourgeois $H$, Extra JM, Serin D, Kerbrat $P$, Machiels JP, Lortholary A, Orfeuvre H, Campone M, Hardy-Bessard AC, Coudert B, Maerevoet M, Piot G, Kramar A, Martin AL, Penault-Llorca F. Trastuzumab for patients with axillary-node-positive breast cancer: results of the FNCLCC-PACS 04 trial. J Clin Oncol 2009;27(36):6129-34.

27. McGuire A, Lowery AJ, Kell MR, Kerin MJ, Sweeney KJ. Locoregiona Recurrence Following Breast Cancer Surgery in the Trastuzumab Era: A Systematic Review by Subtype. Ann Surg Oncol 2017;24(11):312432.

28. Horton JK, Jagsi R, Woodward WA, Ho A. Breast Cancer Biology: Clinical Implications for Breast Radiation Therapy. Int J Radiat Oncol Biol Phys 2018;100(1):23-37.

29. Lanning RM, Morrow M, Riaz N, McArthur HL, Dang C, Moo TA, ElTamer M, Krause K, Siu C, Hsu M, Zhang Z, Pei X, McCormick B, Powell SN, Ho A. The Effect of Adjuvant Trastuzumab on Locoregional Recurrence of Human Epidermal Growth Factor Receptor 2-Positive Breast Cancer Treated with Mastectomy. Ann Surg Oncol 2015;22:2517-25.

30. Adra J, Lundstedt D, Killander F, Holmberg E, Haghanegi M, Kjellén E, Karlsson P, Alkner S.Distribution of locoregional breast cancer recurrence in relation to postoperative radiation fields and biological subtypes. Int J Radiat Oncol Biol Phys 2019;105(2):285-95.

31. Schettini F, Buono G, Cardalesi C, Desideri I, De Placido S, Del Mastro L. Hormone Receptor/Human Epidermal Growth Factor Receptor 2-positive breast cancer: Where we are now and where we are going Cancer Treat Rev 2016;46:20-26.

32. Aalders KC, Postma EL, Strobbe LJ, van der Heiden-van der Loo M, Sonke GS, Boersma LJ, van Diest PJ, Siesling S, van Dalen T. Contemporary Locoregional Recurrence Rates in Young Patients With Early-Stage Breast Cancer. J Clin Oncol 2016:34(18):2107-14.

33. Kim MM, Dawood S, Allen P, Sahin AA, Woodward WA, Smith BD, Strom EA, Hunt KK, Meric-Bernstam F, Gonzalez-Angulo AM, Buchholz TA. Hormone receptor status influences the locoregional benefit of trastuzumab in patients with nonmetastatic breast cancer. Cancer 2012;118(20):4936-43. 
34. Abi Jaoude J, de Azambuja E, Makki M, Tamim H, Tfayli A, Geara F, Piccart M, Poortmans P, Zeidan YH. Post-Mastectomy Radiation Therapy in Human Epidermal Growth Factor Receptor 2 Positive Breast Cancer Patients: Analysis of the HERA Trial. Int J Radiat Oncol Biol Phys 2020;106(3):503-10.

35. Bellon JR, Guo H, Barry WT, Dang CT, Yardley DA, Moy B, Marcom PK, Albain KS, Rugo HS, Ellis M, Wolff AC, Carey LA, Overmoyer BA, Partridge AH, Hudis CA, Krop I, Burstein HJ, Winer EP, Tolaney SM. Local-regional recurrence in women with small node-negative, HER2-positive breast cancer: results from a prospective multi-institutional study (the APT trial). Breast Cancer Res Treat 2019;176(2):30310.

36. Chen X, Ma N, Zhou Z, Wang Z, Hu Q, Luo J, Mei X, Yang Z, Zhang L, Wang X, Feng Y, Yu X, Ma J, Guo X. Estrogen receptor mediates the radiosensitivity of triple-negative breast cancer cells. Med Sci Monit 2017;23:2674-83.

37. Giordano SH, Temin S, Kirshner JJ, Chandarlapaty S, Crews JR, Davidson NE, Esteva FJ, Gonzalez-Angulo AM, Krop I, Levinson J, Lin NU, Modi S, Patt DA, Perez EA, Perlmutter J, Ramakrishna N, Winer EP. American Society of Clinical Oncology. Systemic therapy for patients with advanced human epidermal growth factor receptor 2-positive breast cancer: American society of clinical oncology clinical practice guideline. J Clin Oncol 2014; 32(19):2078-99.

38. Leone JP, Leone J, Zwenger AO, Vallejo CT, Leone BA. Prognostic Significance of Tumor Subtypes in Women With Breast Cancer According to Stage: A Population-based Study. Am J Clin Oncol 2019;42(7):588-95.

39. Swisher SK, Vila J, Tucker SL, Bedrosian I, Shaitelman SF, Litton JK, Smith BD, Caudle AS, Kuerer HM, Mittendorf EA. Locoregional Control According to Breast Cancer Subtype and Response to Neoadjuvant Chemotherapy in Breast Cancer Patients Undergoing Breast-conserving Therapy. Ann Surg Oncol 2016;23:749-56.

40. Kolarova I, Vanasek J, Odrazka K, Melichar B, Ryska A, Petera J, Vosmik $M$, Dolezel $M$. Therapeutic significance of hormone receptor positivity in patients with HER-2 positive breast cancer. Biomed Pap Med Fac Univ Palacky Olomouc Czech Repub 2019;163(4):285-92.
41. Harbeck N. Neoadjuvant treatment of HER2-positive breast cancer: should therapy differ based on hormone receptor status? Ther Adv Med Oncol 2018;10:1-3.

42. Bellon JR. Regional noda lirradiation in the anti-HER2 era. J Natl Cancer Inst 2017;109(8).

43. Halyard MY, Pisansky TM, Dueck AC, Suman V, Pierce L, Solin L, Marks L, Davidson N, Martino S, Kaufman P, Kutteh L, Dakhil SR, Perez EA. Radiotherapy and adjuvant trastuzumab in operable breast cancer: tolerability and adverse event data from the NCCTG Phase III Trial N9831. J Clin Oncol 2009;27(16):2638-44.

44. Jacob J, Belin L, Pierga JY, Gobillion A, Vincent-Salomon A, Dendale $R$, Beuzeboc P, Campana F, Fourquet A, Kirova YM. Concurrent administration of trastuzumab with locoregional breast radiotherapy: long-term results of a prospective study. Breast Cancer Res Treat 2014; 148(2):345-53.

45. Chan A, Moy B, Mansi J, Ejlertsen B, Holmes FA, Chia S, Iwata H, Gnant M, Loibl S, Barrios CH, Somali I, Smichkoska S, Martinez N, Alonso MG, Link JS, Mayer IA, Cold S, Murillo SM, Senecal F, Inoue K, RuizBorrego M, Hui R, Denduluri N, Patt D, Rugo HS, Johnston SRD, Bryce R, Zhang B, Xu F, Wong A, Martin M; ExteNET Study Group. Final Efficacy Results of Neratinib in HER2-positive Hormone Receptorpositive Early-stage Breast Cancer From the Phase III ExteNET Trial. Clin Breast Cancer 2020 Oct 6:S1526-8209(20)30258-5. doi: 10.1016/j.clbc.2020.09.014 Epub ahead of print

46. Bartelink $H$, Maingon $P$, Poortmans $P$, Weltens $C$, Fourquet $A$, Jager J, Schinagl D, Oei B, Rodenhuis C, Horiot JC, Struikmans $H$, Van Limbergen E, Kirova Y, Elkhuizen P, Bongartz R, Miralbell R, Morgan D, Dubois JB, Remouchamps V, Mirimanoff RO, Collette S, Collette L; European Organisation for Research and Treatment of Cancer Radiation Oncology and Breast Cancer Groups. Whole-breast irradiation with or without a boost for patients treated with breastconserving surgery for early breast cancer: 20-year follow-up of a randomised phase 3 trial. Lancet Oncol 2015;16(1):47-56.

47. Kirova YM, Loap P, Fourquetet A. Benefit of Post Mastectomy Radiation Therapy (PMRT) in Node-Positive, HER2-Positive Patients With Breast Cancer Receiving Anti-HER2 Treatments. Int J Radiat Oncol Biol Phys 2020;106(3):511-13. 